\title{
Dynamical friction on cold fractal gas clouds. Applications to disc formation
}

\author{
B. Semelin ${ }^{1}$ and F. Combes ${ }^{2}$ \\ 1 Department of Physics, Waseda University, Oh-kubo, Shinjuku-ku, Tokyo 169-8555, Japan \\ 2 Observatoire de Paris, DEMIRM, 61 Av. de l'Observatoire, 75014 Paris, France
}

Received 7 August 1999 / Accepted 18 February 2002

\begin{abstract}
It is likely that cold molecular clouds form at high redshift, before galaxies. Considering these cold clouds instead of hot homogeneous gas as the main baryonic component of proto-galactic halos may affect several issues during galaxy formation. In particular, the baryonic matter loses angular momentum through dynamical friction on the dark matter halo. In numerical simulations using hot gas for baryonic matter, this gives rise to the so-called angular momentum problem. In this work, we study the dynamical friction exerted on cold fractal gas by a collisionless background (dark matter) through high-resolution numerical simulations. First, we find that, for values of the parameters relevant during galaxy formation, the friction does not alter the morphology of the fractal, which is mainly driven by internal dynamics. Then, we show that the presence of substructures and inhomogeneities in a body has little effect on the global value of the friction. Parameters such as branching ratio and fractal dimension also have little effect. In fact, we find that the main effect comes from the deformations and fluctuations of the fractal structure in cold gas clumps. If the deformation time is of the same order of magnitude or shorter than the typical build-up time of the friction, the friction is weakened. We argue that this effect is relevant for galaxy formation, and that the angular momentum problem should not be worsened by including the small scale inhomogeneities of the cold gas which occur at a resolution out of reach of present simulations.
\end{abstract}

Key words. methods: numerical - galaxies: formation

\section{Introduction}

Our goal in this work is to investigate the consequences of including cold fractal gas in galaxy formation, with a focus on the angular momentum problem.

In the context of galaxy evolution, the importance of considering a multiphase medium for a realistic description of the interstellar medium was first established by Cox \& Smith (1974) and McKee \& Ostriker (1977). They showed that the temperature range relevant to galactic gas dynamics extends from $10 \mathrm{~K}$ up to $10^{6} \mathrm{~K}$. As for the density range, it extends from $10^{-2} \mathrm{~cm}^{-3}$ in hot regions to possibly $10^{6} \mathrm{~cm}^{-3}$ or more in the cores of molecular clouds (see, e.g., Faison et al. 1998 for detection of small dense structures). At each end of these ranges, hot plasma and cold molecular clouds are driven by very different and complex physical processes, and cannot be numerically described in a single phase. For examples of simulations with multiphase descriptions of the interstellar medium on full galactic scale see Rosen \& Bregman (1995), Gerritsen \& Icke (1997) and Wada \& Norman (1999). These works

Send offprint requests to: B. Semelin,

e-mail: semelin@gravity.phys.waseda.ac.jp focus on star formation and interstellar medium morphology, but not on galaxy formation.

Until now, most numerical studies of galaxy formation have considered a single gas phase, treated through $\mathrm{SPH}$ dynamics, cooling being artificially stopped at $10^{4} \mathrm{~K}$ (e.g., Weil et al. 1998; Thacker \& Couchman 2000 or Kay et al. 2000). Some authors have considered simple multiphase models (Yepes et al. 1997; Hultman \& Pharasyn 1999) including cold gas. However, they chose to run simulations on scales of several Mpc, which limits the resolution to $\sim 1 \mathrm{kpc}$. Important differences in the physics of cold and hot gas appear below this scale. This is the case for the fractal structure of cold gas clouds, which we focus on in this work.

In simulations of galaxy formation, one of the main difficulties arises from the "angular momentum problem". During infall, the gas loses angular momentum through dynamical friction on the surrounding dark matter halo: the resulting angular momentum of the disc is too small by a factor of 10 compared to typical values for presentday galaxies (Navarro et al. 1995). Various solutions have been investigated to alleviate the problem. For example, Navarro \& Steinmetz (1997) used a photo-ionizing UV 
background to heat the gas in order to limit the collapse of the disc. They found out however that this actually exacerbates the angular momentum problem, since UV heating mostly prevents late infall of matter, which otherwise supplies most of the angular momentum. Along the same line, Weil et al. (1998) showed that the angular momentum problem can be avoided if the gas is prevented from cooling at early epochs and until $z=1$. However, they do not specify what process is responsible for suppressing the cooling. Dominguez-Tenreiro et al. (1998) included simple star formation without feedback. They showed that the formation of a stellar bulge stabilizes the disc and reduces the infall of gas, thus limiting the loss of angular momentum. Thacker \& Couchman (2000) implement feedback from supernovae in galaxy formation. They find that the final angular momentum can be increased through feedback, but this effect may be insufficient to reach observed values. Consequently, the solution of the problem is still a matter of debate. Moreover, the mass resolution is at best $10^{6}$ solar masses per particle in usual galaxy formation simulations. This is not enough to take into account the fractal structure of cold gas and how dynamical friction acts on it. A local study of this phenomenon is needed.

Our approach is to consider the possibility of disc formation by the accretion of cold fractal gas. It is likely that clumps of molecular gas and GMCs form very early, even before galaxies (Combes \& Pfenniger 1998). The formation of such structures requires two conditions: efficient early cooling leading to quasi-isothermal collapse of primordial density inhomogeneities and inefficient star formation. Indeed, the so-called over-cooling problem is actually a problem only if the cooling of the gas results in overproduction of stars at high redshift, which is inconsistent with observations. Evidence for both factors is presented in Combes \& Pfenniger (1998) and references therein. In the formation of discs from cold fractal gas, one essential process to study is the loss of angular momentum through dynamical friction.

Dynamical friction is a complex non-linear phenomenon, and it is a challenge to predict its effect on a fractal medium. Chandrasekhar (1943) gives the first evaluation of the friction on a test point-particle in a non self-gravitating background. If $M$ is the mass of a test particle, $v_{M}$ its velocity, $m$ the mass of the background particles and $f\left(v_{m}\right)$ an isotropic distribution function for background particle velocities, the test particle velocity changes according to

$\frac{\mathrm{d} v_{M}}{\mathrm{~d} t}=-16 \pi^{2} G^{2} \ln \Lambda m(M+m) \int_{0}^{v_{M}} f\left(v_{m}\right) v_{m}^{2} \mathrm{~d} v_{m} \frac{v_{M}}{v_{M}^{3}}$

where

$\Lambda=\frac{b_{\max } V_{0}^{2}}{G(M+m)}$.

Here $b_{\max }$ is the maximum impact parameter (often the typical size of the background medium) and $V_{0}$ is the typical velocity difference between background and test particle. There is an amount of uncertainty when assigning a value to $\Lambda$ for a given physical application. In the case of an extended body, Chandrasekhar's formula overestimates the friction since background particles with small impact parameters are not as deflected as in the case of a point test particle. For an extended body, it is possible to define a minimum impact parameter $b_{\min }$ and redefine the Coulomb parameter $\Lambda$ :

$$
\Lambda=\frac{b_{\max }}{b_{\min }}
$$

(see Binney \& Tremaine 1987 for more details). In the Chandrasekhar definition, dynamical friction is an acceleration. In this work, we will measure the dynamical friction on a given body as the net acceleration from gravitational forces exerted on this body by the background. We will refer to the norm of this acceleration as the intensity of the friction.

Binney \& Tremaine (1987) review various applications of dynamical friction. Important issues have been to determine whether the effect is local or global, and whether the self-gravity of the response is important or not. Chandrasekhar's approach has a pure local formulation, which does not explain the friction at a distance (Leeuwin \& Combes 1997). This is essential in the braking of two interacting galaxies, for example. In the case of a sinking satellite, including the barycentric motion of the primary body which acts as a background significantly reduces the dynamical friction (Weinberg 1989). Including also the self-gravitation of the primary body does not appear to produce large corrections (Bontekoe \& van Albada 1987; Zaritsky \& White 1988). Another important point, rarely taken into account, is the tidal deformation of the secondary component, the component moving among the primary (background) particles. Since the friction is due to the deformation of the interacting bodies, the freezing of the smallest one leads to an underestimate of the friction; indeed it is the one which actually undergoes the largest deformation (cf. Prugniel \& Combes 1992). The Chandrasekhar formula can be used in many circumstances for dimensional relevance, but its quantitative value can be off by more than an order of magnitude, and this uncertainty is hidden in the Coulomb parameter $\Lambda$ (e.g. Bontekoe \& van Albada 1987). Most frequently, a collisionless background is assumed to estimate the friction, although recently Ostriker (1999) has estimated that the friction against a gaseous medium (collisional) is much stronger. Her treatment is from linear perturbation theory and does not take the self-gravity of the wake fully into account.

Our aim is to establish whether new parameters affect the intensity of the friction when it acts on a fractal body. We consider only a collisionless background, i.e. composed of dark matter particles. Our approach is through numerical simulations.

In Sect. 2 we briefly present the code and numerical methods. In Sects. 3-5 we present the results from our simulations. We give our conclusions in Sect. 6 . 


\section{Numerical techniques and general setting}

\subsection{The code}

The code used to run the simulations is a parallel version of the tree-code (Barnes \& Hut 1986) running on a Cray T3E at IDRIS. The present version is a MPI-MPI2 adaptation of the original PVM-SHMEM version developed by Ninin (1999). The code uses a binary tree technique and the associated natural domain decomposition (Ninin 1999). All simulations are run with an opening angle $\theta=0.8$, governing the use of multipole expansion for a group of particles, or their splitting in sub-groups. This version of the code uses a single time-step. Our simulations require two types of particles: dissipative gas particles and collisionless dark matter particles. Dissipation, included in the simulations of Sect. 3 only, is implemented by inelastic collisions, using the sticky-particle model (Levinson \& Roberts 1981). We use periodic boundary conditions throughout the paper, the potential being computed with Ewald method (Ewald 1921). No cosmological expansion effect is included.

\subsection{Geometry and scales: Simulation setup}

Our goal is to reassess the angular momentum problem when the basic proto-galactic material is a distribution of cold fractal gas clouds. Studying such a system presents a major difficulty from the computational point of view: the relevant scale range goes from $10^{-4} \mathrm{pc}$ (the size of the smallest structures in giant molecular clouds, Faison et al. 1998) to $10^{5} \mathrm{pc}$, a possible value for the size of the local proto-galactic halo. We have to reduce this range drastically in the simulations. The fractal structure of the cold gas mostly appears at scales smaller than $1 \mathrm{kpc}$; at larger scales it is broken by the shear arising from the global geometry of the system (for example in differentially rotating discs). Is dynamical friction on structures at this scale likely to be a large part of the overall loss of angular momentum? It depends on the merging history of the forming galaxy. Indeed, dynamical friction is proportional to the mass of the body. So, if the galaxy formed through the merging of a few large bodies, the friction at small scales will not play a large part in the total angular momentum loss. However, if the merging history is quiet, and the galaxy acquires matter mainly through accretion, then small-scale friction must be evaluated as it may play an important part in angular momentum loss. Consequently, we focus on the scale of a single, or a few molecular clouds; that is, a value of the order of $1 \mathrm{kpc}$ for the simulation box size. Accordingly the baryonic mass in the box will be around $10^{7}$ solar masses, and the dynamical time around $10 \mathrm{Myr}$. In the simulations these quantities are normalized to 1 .

The main effect of this zoom-in is that the loss of angular momentum will actually be studied as a loss of linear momentum, as the difference in in the bulk motion of the dark matter halo and the proto-galactic gas cloud translates, on small scales, into a uniform drift between the two components. Since we do not take the global dynamics into account, the drift between dark matter and gas must be artificially sustained. The drifting speed must be chosen of the same order as the virial velocity of the system ( $100 \mathrm{~km} \mathrm{~s}^{-1}$ for a typical galaxy).

At the chosen scales (between $10 \mathrm{pc}$ and $1 \mathrm{kpc}$ ), the gas has already collapsed in dense clumps, raising the density ratio between gas and dark matter above one.

\subsection{Periodicity and reference frame}

Since we set the simulation box size to a sub-galactic scale, two questions arise: how is the matter outside the simulation box taken into account, and what kind of reference frame is associated with the simulation box, given the globally rotating nature of the proto-galactic cloud?

First, let us sum up our approach for the forming galaxy. We consider a large number of fractal clumps of cold gas, accreting to form a disc, and we investigate the effect of dynamical friction over scales smaller than $1 \mathrm{kpc}$. In this picture, the dynamical friction on each clump is local.

The presence of matter (both gas and dark matter) outside the box is taken into account by using periodic boundary conditions. This recreates the distribution of clumps, albeit with an artificially regular pattern.

For a fully self-consistent simulation, a non-inertial frame should be used. There is a well-known procedure, for example, when simulating a small piece of a rotating disc (Wisdom \& Tremaine 1988; Semelin \& Combes 2000). However, we believe that in our study, Coriolis forces and drag from differential rotation are secondary processes since the dynamical friction arises from local gravitational effects. The centrifugal force is of course balanced by the global gravitational field of the forming galaxy. Consequently we use an inertial frame.

\section{Dynamical simulations}

\subsection{Initial conditions}

In this first set of simulations, we study the general case, a collapsing gas medium within a drifting dark matter background.

The average ratio of the density of the gas to the density of the dark matter is set to 9 . We use 64000 particles for each component.

We want a homogeneous initial gas density field with fluctuations whose power spectrum follows a power law of index -2 . Using the continuity equation in the linear regime, we can find the correct power law for the power spectrum of the velocity field which produces the desired density fluctuations. We use this field to assign velocities to the particles initially distributed on a regular grid. The velocity field amplitude is set such that the simulation box includes several tens of Jeans masses for the gas, allowing for efficient fragmentation. Furthermore, the gas 

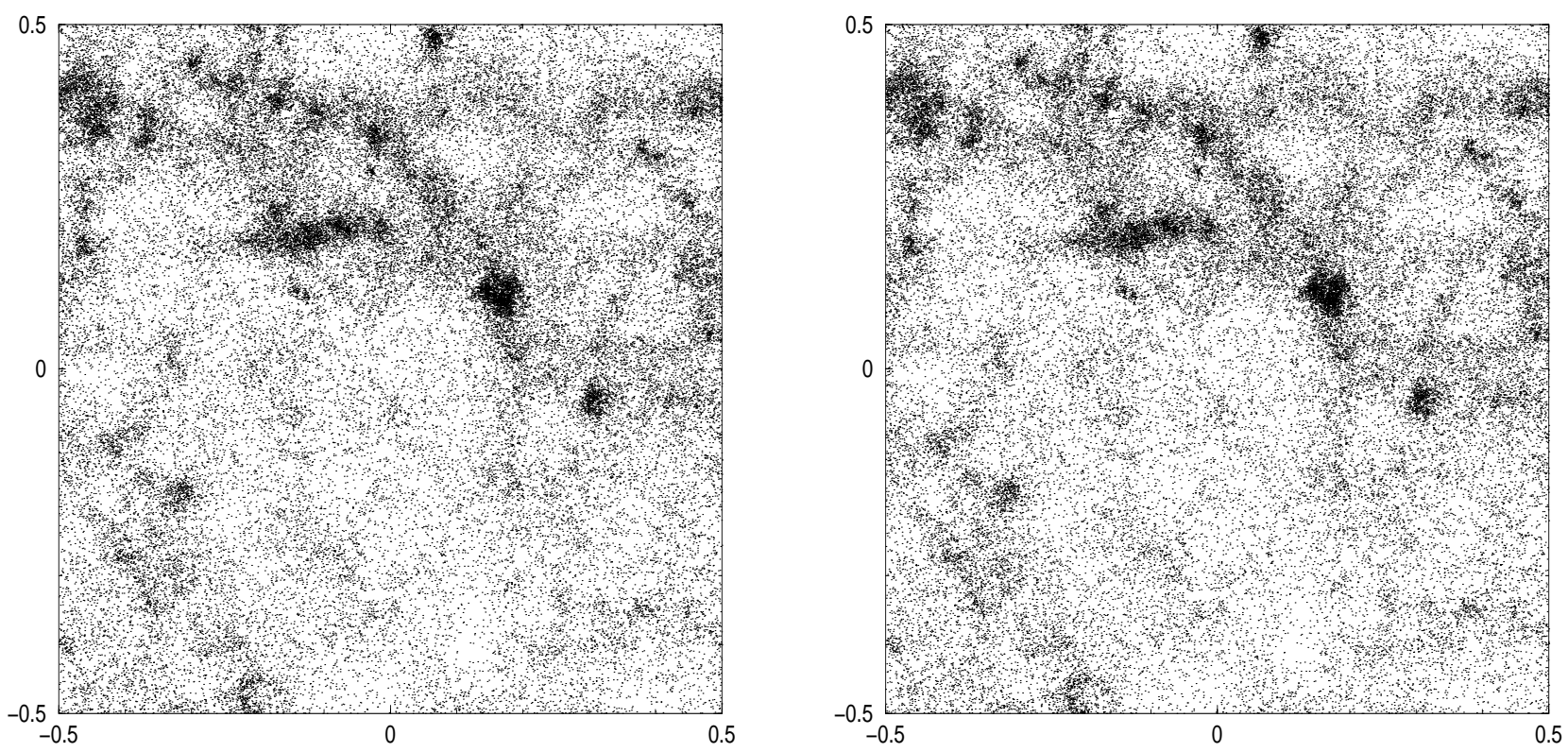

Fig. 1. Plots of the gas component (64000 particles) after 0.45 dynamical times. On the left, the dark matter background (also 64000 particles, not plotted) has no initial drift. On the right, the initial drift is $v_{x}=0.5, v_{y}=0.2$ and $v_{z}=0.2$, creating a dynamical friction. The density fields of the gas are remarkably similar.

undergoes dissipation in inelastic collisions. The dissipation is strong enough to absorb the potential energy released in the collapse (isothermal collapse condition). This means that at least half of the kinetic energy of the gas is dissipated within 1 free-fall time.

The dark matter density field is homogeneous, and the thermal motion strong enough to prevent collapse on the box scale. We run two simulations. In the second simulation, an additional uniform velocity field is added to the dark matter to account for the drift between dark matter and gas responsible for the dynamical friction. Initially the dark matter, not the gas, is drifting since our frame is attached to the gaseous component. Consequently we expect the dark matter to slow down and the gas to accelerate until the drift is canceled as an effect of dynamical friction. The global drift velocity of the dark matter is chosen of the same order as the ratio between box size over dynamical time ( 1 in the simulation dimensionless units, and of the order of $100 \mathrm{~km} \mathrm{~s}^{-1}$ in physical units). This is also the order of magnitude of the virial speed for the dark matter component. According to Chandrasekhar's formula, it is the range where the friction is maximal (see also Ostriker 1999).

We run the two simulations over 1 dynamical time (typically $10 \mathrm{Myr}$ ) using 400 time-steps, keeping energy conservation within $1 \%$.

\subsection{Results}

Figure 1 shows plots of the gas component after $0.45 \mathrm{dy}$ namical time for both simulations. The plots are remarkably similar. To quantify this, we have binned the density field on a $16^{3}$ grid (each cell contains 15.6 gas particles on average). Then, we define the usual mathematical norm:

$$
\|\rho\|=\left(\int \rho^{2} \mathrm{~d} \boldsymbol{x}\right)^{\frac{1}{2}}
$$

If we call $\rho_{0}$ the initial density field, $\rho_{1}$ the final density field without dynamical friction, and $\rho_{2}$ the final density field with dynamical friction, we have computed:

$$
\begin{aligned}
& \frac{\left\|\rho_{2}-\rho_{0}\right\|}{\left\|\rho_{0}\right\|}=1.90 \ldots \\
& \frac{\left\|\rho_{2}-\rho_{1}\right\|}{\left\|\rho_{0}\right\|}=0.091 \ldots
\end{aligned}
$$

For comparison we started from different initial conditions and called $\rho_{3}$ the collapsed state at $t=0.45$. We get:

$$
\frac{\left\|\rho_{3}-\rho_{1}\right\|}{\left\|\rho_{0}\right\|}=2.52 \ldots
$$

This shows that the two configurations in Fig. 1 are indeed similar. The effect of dynamical friction on the gas component is obviously negligible at this stage. Later times in the integration do not show much more effect, while the fractal structure begins to be broken by the lack of energy input and merges to a few "black holes". This does not mean that friction does not operate.

Figure 2 shows a noticeable effect on the dark matter, which loses a sizeable fraction of its initial momentum in the simulation including a drift of the background over $0.8 t_{\mathrm{dyn}}$. At $t=0.45 t_{\mathrm{dyn}}$, however, the dark matter has lost only a few percent of its momentum. The dynamical 
friction acts, but not soon enough to affect the collapse of the gas. On the chosen scale, the dynamics of the gas cloud is dominated by its self-gravity which acts on much shorter time scales than the dynamical friction. Therefore, to study the effect of the dynamical friction, it is necessary to explore longer time scales in a quasi-stationary regime.

In this regard, the main difficulty is to obtain a longlived fractal structure self-consistently. It requires full modeling of the interstellar medium physics over a large range of scales, including complex processes for the dissipation and energy input. See, for example, Semelin \& Combes (2000) and Huber \& Pfenniger (2001). Work is still going on in this area. In this work, we will follow a simple approach. We freeze the fractal and run a semidynamical simulation. Since the internal dynamics of the fractal are dominated by its self-gravity, dynamical friction should not strongly affect the morphology of the fractal, and little physical significance is lost in a semidynamical simulation, as far as evaluating the intensity of the dynamical friction is concerned. In Sect. 5 we will follow another possible approach: imposing artificial dynamics on the fractal.

\section{Dynamical friction on a frozen fractal body}

In this section we focus our attention on an individual structure in the cold gas halo. This structure may be considered either as an individual giant molecular cloud or as a substructure embedded in a larger fractal structure. In both cases periodicity provides the neighboring gas structures. In physical systems, the slow decay of the orbit of the structure during disc formation sustains the drift between gas and dark matter, and produces a local quasistationary state for the structure. Consequently, in the simulation we can attach the frame to the center of gravity of the structure and get an almost inertial frame (centrifugal force is balanced by gravity and Coriolis forces are negligible). Since our simulation is local, the drift has to be artificially sustained.

In the following simulations, a rigid, motionless multiparticle body of typical radius 0.1 (box size equal 1) and mass 0.2 (total mass equal 1) is subject to dynamical friction from a collisionless background. We give to this central body an artificial fractal structure (described below). This permits a meaningful comparison with an equivalent homogeneous body and a fine control over the characteristic parameters of the fractal structure. Because of the periodic boundary conditions the maximal impact parameter is intentionally much smaller than in usual studies of the dynamical friction: around 3 to 4 times the value of the minimal impact parameter depending on exact definitions. This value is consistent with both possibilities for the central body: if it is a giant molecular cloud, neighboring clouds are likely to be close by as observed in galactic discs, and if it is a substructure within a fractal clump, typical substructure separation is also quite small.

The central body is much more dense (50 times) than the background dark matter, as can be expected for a

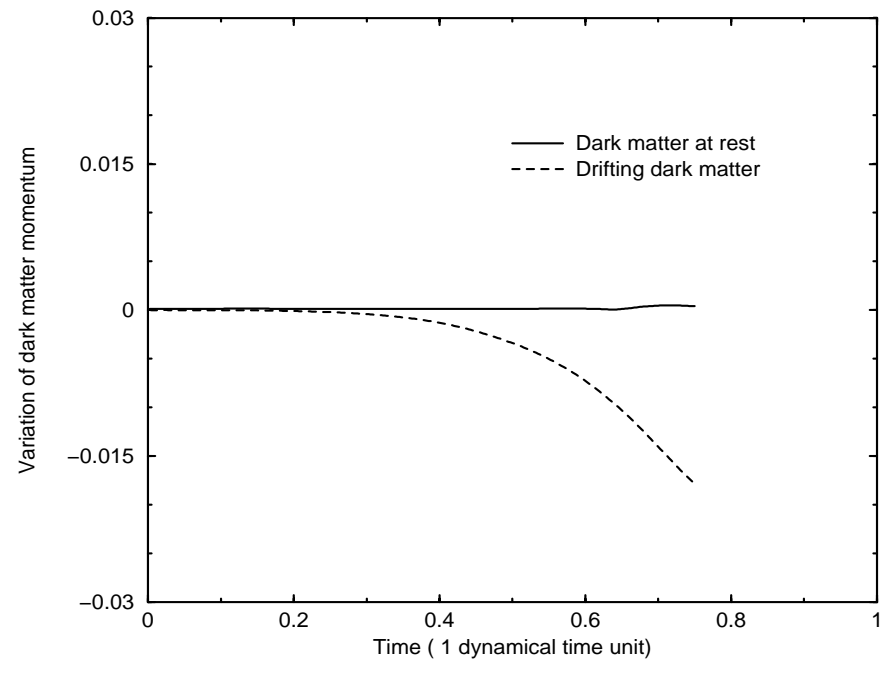

Fig. 2. Variation of the total momentum of dark matter as a function of time, with or without dynamical friction (i.e. with or without a velocity drift between gas and dark matter). In the case where there is a velocity drift between the two components, the dark matter total momentum shows a variation, giving the order of magnitude of the friction. The fact that the variation is a decrease is only due to the choice of reference frame of the gas, where the dark matter is moving and the gas is at rest. In the galaxy's environment, the gas is in motion relative to the dark matter, whose angular momentum increases.

dense molecular cloud. The total integration time of the simulations is about 40 times the dynamical time the central body would have if it could evolve.

\subsection{Building an artificial fractal body}

There are many possible procedures for building a fractal set of point particles. A simple approach is to build a hierarchy of structures recursively. In this work we use a hierarchy of spheres. At level 0 of the hierarchy, the basic sphere has radius $r_{0}$. Then we define two parameters. The branching ratio $a$ specifies the number of sub-spheres we will include in each sphere. The scale ratio $b$ defines the radii of the sub-spheres: at level $n$ spheres will have a radius $r_{0} b^{-n}$. To avoid a strong scale periodicity (see appendix A in Semelin \& Combes 2000), we introduce a variance when assigning the radius of each sub-sphere, equal to $\frac{1}{3}$ of the average radius at this level. Given these two parameters, $a$ and $b$, the recursive procedure is the following: each sphere is subdivided into $a$ sub-spheres of radii $\sim \mathrm{b}$ time smaller. We place the center of each sub-sphere at random on the surface of the initial sphere. Different choices are possible here. For example we could use a probability function $\left(\frac{1}{r^{2}}\right.$, constant, etc. $)$ to distribute the centers of the sub-spheres inside the sphere. However, the branching ratio being usually smaller than 10 , the statistical realization of the probability law is noisy, and different laws produce similar fractals. When the center and radii of the spheres at the last level of the hierarchy have been 

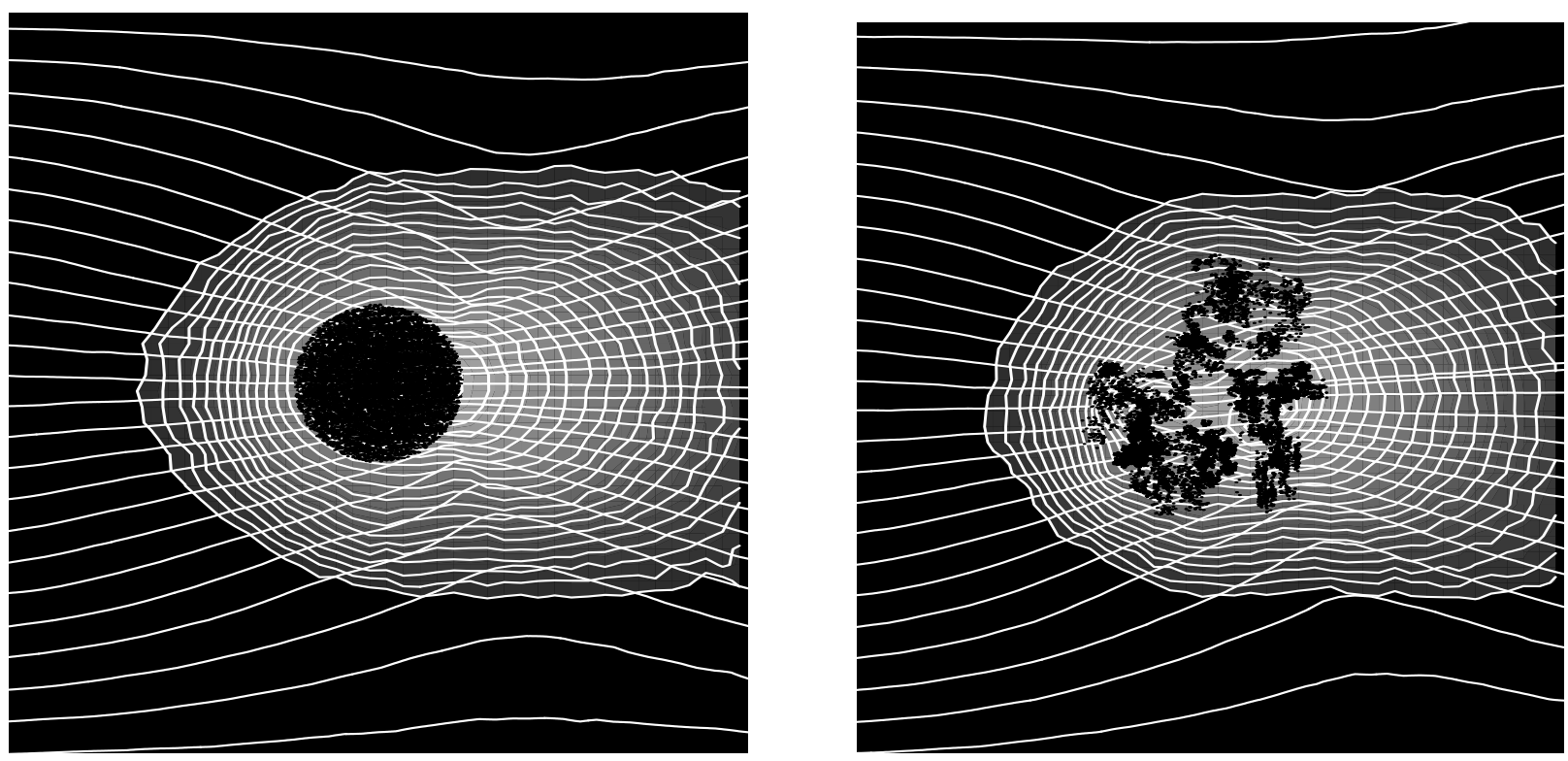

Fig. 3. Stationary state dynamical friction on two different bodies. The density contours and streamlines for a slice of the background are plotted, smoothed over one crossing time. The body on the left is a homogeneous sphere. The body on the right is a 5 level artificial fractal with dimension 2 .

defined, we distribute particles in these spheres evenly. We divide each structure into substructures of equal mass. Here again different choices can be made, for example using a Gaussian probability function to determine the mass of each sub-sphere. Following this procedure, we build a fractal whose fractal dimension is defined by $a$ and $b$ according to: $D_{f}=\frac{\ln (a)}{\ln (b)}$.

\subsection{Conditions of the simulation}

The central body is typically made of 16000 particles. Its structure ranges from a simple spherical distribution to an artificial fractal based on spheres with a 5 level hierarchy of structures, built using the procedure described in Sect. 4.1. Since the body is frozen, velocities are zero at all times.

The drifting dark matter background is made of collisionless particles. Two sets of simulations have been run, the first with 30000 background particles, the second with 1 million background particles. Initially, particles are distributed homogeneously with enough thermal motion to prevent collapse, and an additional drifting speed in the $x$ direction with a value of 0.5 . To reach a stationary state, it is necessary to sustain the drifting speed of the background against slowdown caused by dynamical friction. This is done in two steps. First, particles exiting the simulation box at $x=0.5$ reenter at $x=-0.5$ with random $y$ and $z$ coordinates, and a regenerated momentum. However, simulations show that this is not enough to reach stationarity. It is necessary to add the second factor, a uniform force field which maintains the overall momentum of the background. The norm of the force is proportional to the difference between the actual background total momentum and the desired value. This field accounts for the external large scale phenomena which sustain the drift. Our simulations run over 2.5 box-crossing times for the background.

\subsection{Morphology of the wake}

Figure 3 shows simulations with a one million particle background, and two different central bodies: a homogeneous sphere and a fractal of dimension $D=1.75$ (typical observed value for the interstellar medium, see Combes 1999), branching ratio $a=7$, and a scale ratio $b=3$. The density and velocity fields of the background are binned on a $32^{3}$ grid, then averaged over one crossing time. Then we compute the density contours and the streamlines of the central slice $(x-y$ plane), and plot them in Fig. 3. The complete central body is projected on the slice. In the case of the fractal body, the density contours of the background show two local maxima, connected to substructures in the fractal. These denote the existence of secondary gravitational wakes around the clumps within the central body. Different slices (not plotted here), centered on different substructures in the $z$ direction show different maxima in the density contours of the background, denoting more "sub-wakes". These secondary wakes underline the fact that temporary structures form in the dark matter around the density structure of the fractal body at all scales. These density structures are connected with perturbations in the velocity field, stirring velocity dispersion at all scales. Confirmation of this effect is shown in Fig. 4, which plots the average difference of the friction intensity between two particles in the central body as a function of their distance. This diagnosis is plotted for both the spherical body and a 5 level fractal. Obviously, in the case of the fractal, the spatial fluctuations of the friction remain 


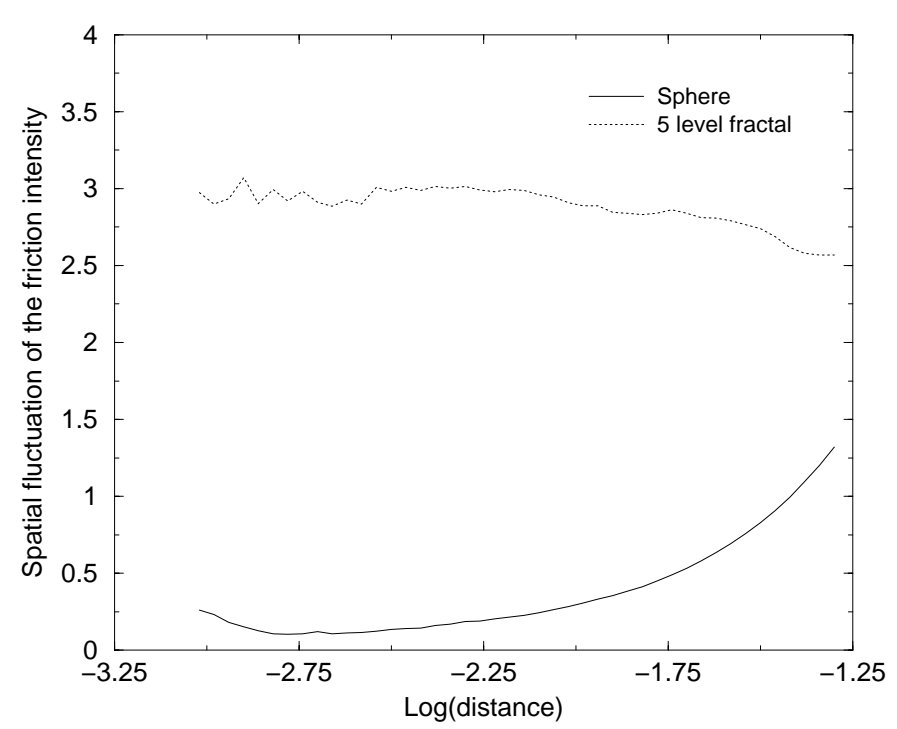

Fig. 4. Fluctuation of the friction between two particles of the central body, as a function of the inter-particle distance. We compute the difference of gravitational force exerted by the background on two particles for every pair of particles in the central body. Then we average the norm of the difference over pairs with the same separation. Plots are given for an homogeneous sphere and a 5 level fractal $(a=7, b=3)$. There are big fluctuations in the case of the fractal. These average out at large scales, where the friction intensity tends to that of an homogeneous sphere.

strong at small scales, whereas they decrease in the case of the sphere. This is again due to the presence of density structures producing secondary wakes at all scales in the case of a fractal body. It is however obvious in Fig. 3 that the large-scale shape of the wake is very similar for the two kinds of bodies.

\subsection{Total intensity of the dynamical friction}

We now assess the influence of the various parameters of the fractal body on the total intensity of dynamical friction, since it could affect the loss of angular momentum in galaxy formation.

The most significant parameter is the scale range of structures present in the fractal. Does the presence of substructures affects the global intensity of the friction? In the case of our artificially constructed fractal, the scale range of the structures is controlled by the number of levels in the hierarchy, from 1, the homogeneous sphere, to 5 levels for a fractal extending over two orders of magnitude in scale.

Figure 5 shows plots of the intensity of the dynamical friction as a function of time. The dynamical friction is evaluated by summing the accelerations of the particles in the central body. The self-gravity of the body cancels out. The two curves correspond to a 1 million and a 30000 particle background. An important noise reduction can be observed, calling for the use of 1 million particle simulations. As can be expected, the observed damped oscillations have

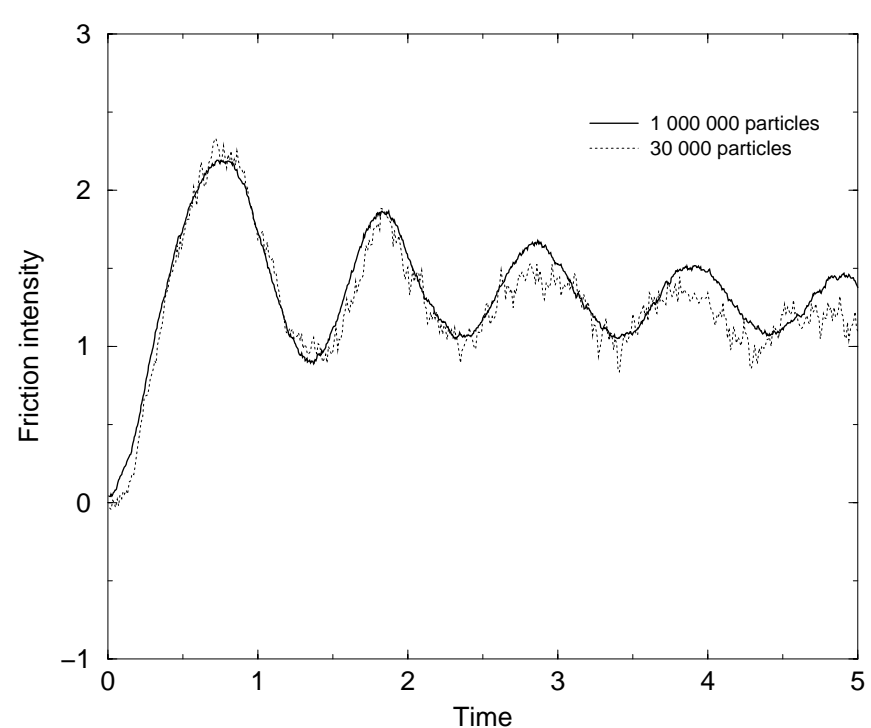

Fig. 5. Total intensity of the dynamical friction for backgrounds with 30000 and 1000000 particles. The curves are not smoothed. The central body is a 2 level "fractal"; that is, a composite object made of 7 spheres of varying radii.

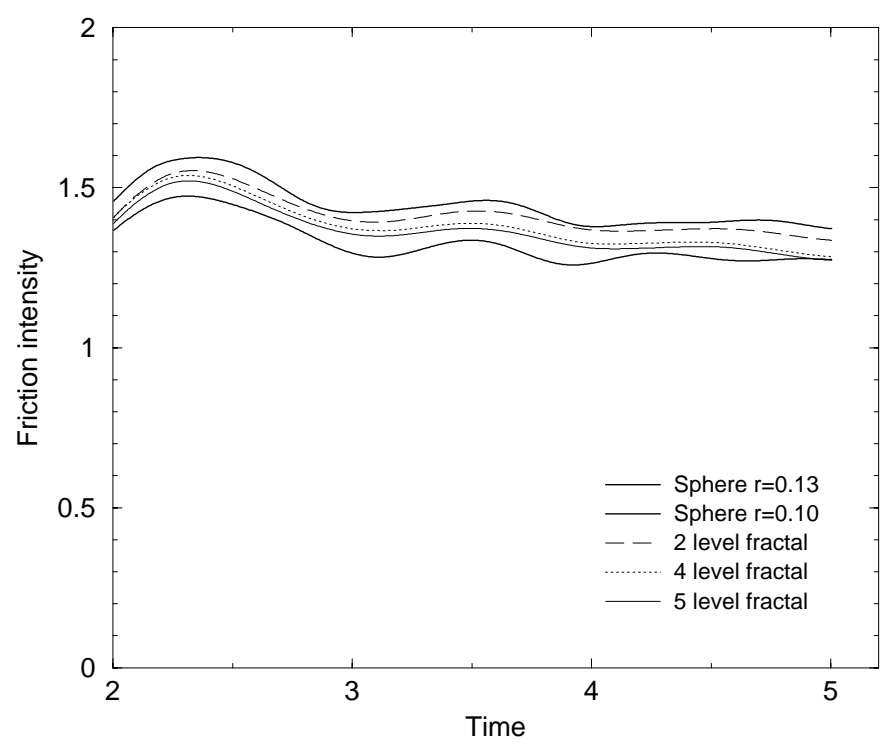

Fig. 6. Total intensity of the friction as a function of time, smoothed over one crossing time. Plots for 2 different spheres, and 3 different fractals $(a=7, b=3)$.

a period similar to the free fall time of the background. We can see that stationarity is not fully reached after 2.5 crossing times. However, because of the computation cost of the 1 million particles simulations, we will be satisfied to evaluate an average value for the friction by smoothing the later parts of the curves.

Figure 6 presents smoothed plots of the intensity of the friction as a function of time for 5 different simulations. All simulations use a 1 million particle background, and extend over 2.5 crossing times with 1000 time steps per simulation. The two thick full lines correspond to spherical central bodies with radii 0.1 and 0.13 , bracketing the values of the effective radii of the other bodies. Indeed, 
when applying the Chandrasekhar formula to an extended body, the effective radius determines the minimal impact parameter, and thus the intensity of the friction. We must keep track of the variation due to this parameter. The thin lines correspond to fractal bodies with 2, 4 and 5 levels in the hierarchy. The obvious conclusion is that the presence of substructures has little effect on the total intensity of the friction. Moreover, we have been able to verify that what little dispersion in the intensity of the friction can be seen between the 2, 4 and 5 level fractal has a simple orgin, for the most part. It can be partly accounted for using Chandrasekhar's formula by a variation of the total effective radius, i.e. a variation in the minimal impact parameter. Let us emphasize again that the ratio of maximal to minimal impact parameter is small, so inhomogeneities in the central body can be felt throughout the simulation box, and this absence of measurable effect was not expected.

Complementary simulations have been run with 30000 particle backgrounds to evaluate the influence of parameters such as the branching rate in the fractal and the value of the fractal dimension. As can be expected from the previous result, no systematic effect has been detected other than from a variation of the minimal impact parameter.

\section{Dynamical friction on a fractal with an artificial dynamics}

In the previous section we established that, beyond its size, the specific spatial structure of a body submitted to dynamical friction has little effect on the intensity of the friction. However, in addition to the fractal structure, cold molecular gas has another important property: the structures evolve and change shape constantly, as a result of dissipation and energy input processes. We will now investigate the impact of this behavior on the dynamical friction.

\subsection{Conditions of the simulations}

As already stated, modeling the long-lived dynamical fractal structure of molecular clouds self-consistently is still a challenge. Since our focus is on dynamical friction, we choose to impose artificial dynamics on the fractal structure. Using the setup described in Sect. 3, we run two simulations, with two different random realizations of the initial conditions. We record the fractal structure of the gas component at $t=0.5 t_{\mathrm{dyn}}$. These two self-consistently built fractal configurations are the basis of our artificial motion.

One of the configurations is used for the initial positions of the gas particles. Then, each particle moves in a straight line with an individual constant speed toward its position in the second fractal. All particles reach the second fractal configuration at the same time. The process is then repeated toward a globally translated version of the first fractal, and so on if necessary. Figure 7 shows the two fractal states and one intermediate state. Although the intermediate state is less clumpy, it retains some definite structures. The fact that the intermediate states do not come closer to homogeneity is due to the relation between the positions of a given particle in the two extremal fractal states. Indeed, the simulations producing these states start from particles on a grid with a specified velocity field. In most cases closeness on the grid implies closeness of the positions in the final fractals. This closeness is retained in part in the transition between the two configurations.

The characteristics of the background are the same as in Sect. 3.

\subsection{Results}

We want to evaluate the effect of a shifting fractal structure on the friction exerted by the background.

First, we run two reference simulations with two different frozen structures: one with the initial, fully fractal configuration and the other with the intermediate, lessclumpy state. Then we run two simulations with an evolving fractal structure, following the procedure described in Sect. 5.1 for the gas motion. We choose two different values, 0.5 and 1 , for the time required to shift from one fully fractal structure to the other. The dynamics of the background is self-consistently computed under the gravitational potential of the gas.

Figure 8 shows plots of the total dynamical friction exerted on the gas by the background for the four simulations. As can be expected, the friction on the frozen lessclumpy intermediate state is weaker than on the frozen, clumpy, initial state. In both cases, the friction builds up until $t=0.5$, then decays because of the heating-up of the wake. By comparison, the friction is weaker for both simulations involving a shifting fractal. Indeed the friction does not have time to build up around clumps as they move, dissolve and collapse constantly. As the curves show, the faster the evolution, the weaker the friction. In the limit of very slow evolution, the friction intensity would reach the same value as for the frozen states.

In actual situations, this effect is relevant if the typical formation time of the wakes, which is of the order of $\frac{b_{\max }}{v_{\mathrm{drift}}}$, is of the same order or longer than the typical evolution time of the structure $\left(b_{\max }\right.$ is the maximal impact parameter and $v_{\text {drift }}$ is the drifting speed between the background and the body). If we consider an average GMC of size $100 \mathrm{pc}$ and speed dispersion $10 \mathrm{~km} \mathrm{~s}^{-1}$, the typical deformation time is $10 \mathrm{My}$. In the case of either galaxy mergers or accretion of cold fractal clumps during disc formation we can evaluate $v_{\text {drift }}=100 \mathrm{~km} \mathrm{~s}^{-1}$ and $b_{\max }$ is a few times the size of the clumps, for example 500 pc. This gives a typical build-up time of the gravitational wakes of $5 \mathrm{My}$. For these values, the weakening of the dynamical friction described above is relevant. The most direct application is to the angular momentum problem in galaxy formation, which will not be worsened by 

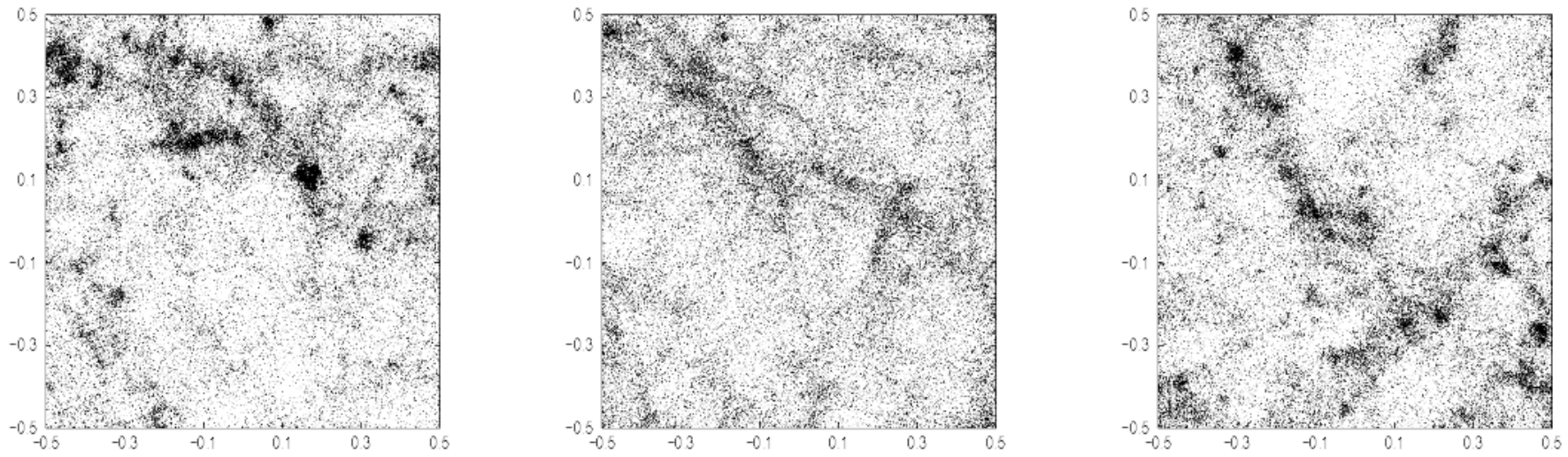

Fig. 7. Plots of gas configurations at different times in the artificial motion. From left to right, the gas follows a linear transformation between two fractal states resulting from self-consistent simulations.

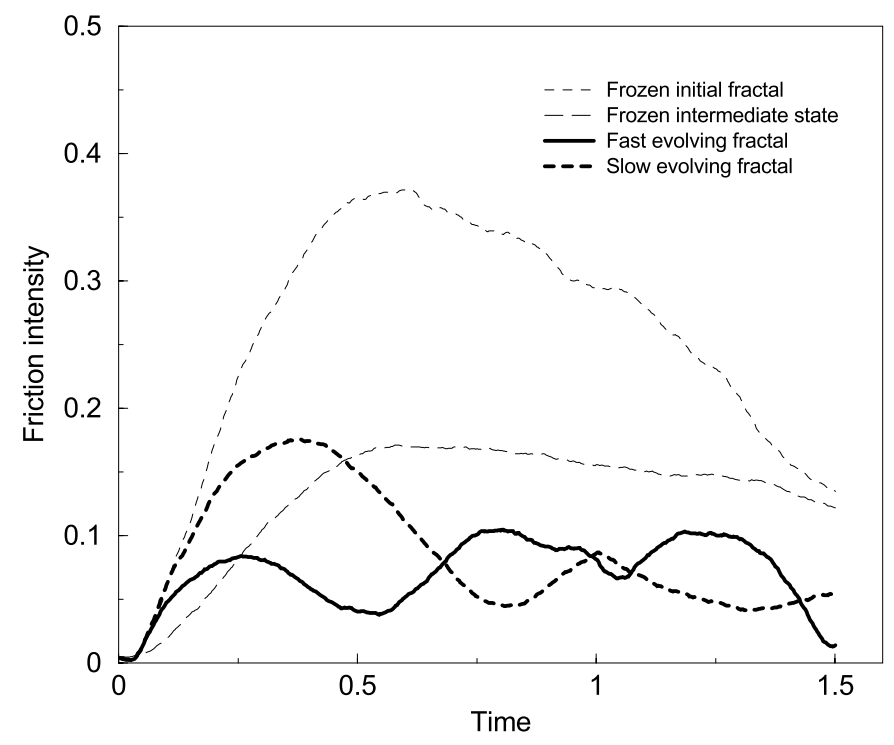

Fig. 8. Time evolution of the dynamical friction in four different cases. Thin lines are for friction on frozen objects, the initial fractal and the intermediate state. Thick lines are for objects evolving from the first fractal, through the intermediate state, to the second fractal, and so on. The duration of the transformation is 0.5 in one case, and 1 in the other.

the presence of fluctuating small scale structures in the cold fractal gas.

\section{Conclusion}

In this work, we have investigated dynamical friction acting on fractal structures such as can be found in molecular clouds. We have argued that this process can be relevant to galaxy formation, and more specifically to the angular momentum problem. Indeed GMCs are likely to form at high $z$ and play an important part in the formation of galactic discs, during which angular momentum is lost through dynamical friction between the gas and the dark matter halo. Due to their different physics, the dissipative gas and the collisionless dark matter particles have different dynamics, and relative bulk motions between these two components are expected to be of the order of the virial velocities in galactic systems. These relative motions should apply to galaxy formation conditions, gravitational collapse or galaxy mergers as well.

We have first established that, for relevant values of the parameters, the fractal structure of the gas is driven by internal processes including self-gravity, and that dynamical friction is not likely to be strong enough to affect GMC morphology. Then we examined if and how GMC morphology could affect the intensity of the friction. Using a frozen central structure, we have established that the presence of inhomogeneities and substructures has little effect on the intensity of the friction. This holds down to small values of the maximum impact parameter, for which inhomogeneities are most likely to affect the global shape of the gravitational wake. What we did find, however, is the presence of secondary wakes connected to substructures. This shows that friction on a fractal body stirs velocity dispersion on all scales in the drifting background.

To complete the study, we have evaluated the response of the friction to changes in the shape of the fractal structure with time, as happens in molecular clouds. We have shown that if the typical formation time of the gravitational wake is longer or of the same order as the deformation time of the structures, the friction is weakened. This effect can have important consequences for the dynamics of galactic discs. If we consider the formation of galactic discs from an assembly of fractal clumps, the weakened friction on the dark matter means a smaller loss of angular momentum for the gaseous disc.

In numerical simulations of galaxy mergers or galaxy formation, particles representing gas clouds have very large mass, due to the lack of resolution and the small number of particles. Typically, a gas cloud is of the order of a GMC mass or bigger (Barnes \& Hernquist 1992). The same is true for particles representing stars, that are as massive as globular clusters. Since the dark matter particles are of comparable mass, friction at small scale cannot be computed and is ignored. However, the friction is important at all scales; and the results presented here show that the fine structure of the interstellar medium does not lead to a worsening of the problem. 
Acknowledgements. Most computations in this work have been realized on the Cray T3E of the CNRS computing center, at IDRIS. The work of B. Semelin was supported by a JSPS grant.

\section{References}

Barnes, J. E., \& Hut, P. 1986, Nature, 324

Barnes, J. E., \& Hernquist, L. 1992, ARA\&A, 30, 705

Binney, J., \& Tremaine, S. 1987, Galactic Dynamics (Princeton PUP)

Bontekoe, T. R., van Albada, T. S. 1987, MNRAS, 224, 349

Chandrasekhar, S. 1943, ApJ, 97, 255

Combes, F., \& Pfenniger, D. 1998, Memorie della Societa Astronomia Italiana, 69, 413

Combes, F. 1999, Proc of The Chaotic Universe, World Scientific Advanced Series in Astrophysics and Cosmology, ed. V. Gurzadyan, Li-Zhi Fang, \& R. Rufini

Cox, D. P., \& Smith, B. W. 1974, ApJ, 189, L105

Domínguez-Tenreiro, R., Tissera, P. B., \& Sáiz, A. 1998, ApJ, 508, L123

Ewald, P. P. 1921, Ann. Phys., 64, 253

Faison, M. D., Goss, W. M., Diamond, P. J., \& Taylor, G. B. 1998, AJ, 116, 2916

Gerritsen, J. P. E., \& Icke, V. 1997, A\&A, 325, 972
Huber, D., \& Pfenniger, D. 2001, A\&A, 374, 465

Hultman, J., \& Pharasyn, A. 1999, A\&A, 347, 769

Kay, S. T., Pearce, F. R., Jenkins, A., et al. 2000, MNRAS, 316,374

Leeuwin, F., \& Combes, F. 1997, MNRAS, 284, 45

Levinson, F. H., \& Roberts, W. W. 1981, ApJ, 245, 465

McKee, C. F., \& Ostriker, J. P. 1977, ApJ, 218, 148

Navarro, J. F., Frenk, C. S., \& White, S. D. M. 1995, MNRAS, 275,56

Navarro, J. F., \& Steinmetz, M. 1997, ApJ, 478, 13

Ninin, S. 1999, Ph.D. Thesis

Ostriker, E. C. 1999, ApJ, 513, 252

Prugniel P., \& Combes, F. 1992, A\&A, 259, 25

Rosen, A., \& Bregman, J. N. 1995, ApJ, 440, 634

Semelin, B., \& Combes, F. 2000, A\&A, 360, 1096

Thacker, R. J., \& Couchman, H. M. P. 2000, ApJ, 545, 728

Wada, K., \& Norman, C. A. 1999, ApJ, 516, L13

Weil, M. L., Eke, V. R., \& Efstathiou, G. 1998, MNRAS, 300, 773

Weinberg, M. 1989, MNRAS, 239, 549

Wisdom, J., \& Tremaine, S. 1988, AJ, 95, 925

Yepes, G., Kates, R., Khokhlov, A., \& Klypin, A. 1997, MNRAS, 284, 235

Zaritsky, D., \& White, S. D. M. 1988, MNRAS, 235, 289 